\title{
Cryo-STEM Imaging of Ribosomes Using the Electron Microscope Pixel Array Detector
}

\author{
Katherine A. Spoth ${ }^{1}$, David A. Muller ${ }^{1,2}$, and Lena F. Kourkoutis ${ }^{1,2}$ \\ 1. School of Applied and Engineering Physics, Cornell University, Ithaca, NY, USA. \\ 2. Kavli Institute at Cornell for Nanoscale Science, Ithaca, NY, USA.
}

Pixelated direct detectors for STEM promise to extend the use of STEM in biology by allowing the collection of the full diffraction pattern at each scan pixel comprising nearly all of the electrons incident on the specimen. This permits imaging of dose-sensitive specimens - difficult with conventional STEM detectors that discard portions of the incident beam to form images. Additionally, these detectors enable advanced imaging methods such as ptychography, which has recently demonstrated resolution beyond the optical limit in a 2D material by utilizing the full range of scattered electrons [1]. In simulations, electron ptychography applied to isolated macromolecules suggest that atomic resolution in $3 \mathrm{D}$ reconstructions may be achievable with far fewer particles compared to single particle EM [2]. So far, however, practicalities of data collection and difficulty in ptychographic reconstructions of comparatively thick biological specimens have precluded an experimental demonstration.

Here, we image purified $80 \mathrm{~S}$ S. cerevisiae ribosomes using the Electron Microscope Pixel Array Detector (EMPAD), a direct detector optimized for STEM through its high dynamic range, singleelectron sensitivity and fast readout speed [3]. One single convergent beam electron diffraction (CBED) pattern from the specimen is shown in Figure $1 \mathrm{~b}$ and illustrates the capabilities of the detector: the entire forward-scattered beam can be imaged without saturating (left) while the low noise allows detection of single electron events at higher scattering angles (right) highlighted by scaling the image intensity.

We employ tilt-corrected bright-field (tcBF-) cryo-STEM imaging, which allows us to collect more than 5 times more electrons than in the conventional BF-STEM detector geometry while retaining coherency. This technique combines images from each pixel of the detector located in the bright field disk after correcting for shifts between the images due to aberrations in the probe. The detection scheme is coherent, as each pixel is small relative to the convergence angle of the probe, producing an image with phase contrast similar to TEM. Calculating the shift between images to sub-pixel resolution encodes information beyond the scan pixel size, which we exploit by up-sampling the images immediately prior to applying the shifts. Resulting images of ribosomes (Figure 1a) show clear detail of the structure and demonstrate good contrast and signal-to-noise, likely a result of the extremely high detection sensitivity of the EMPAD. Here, the scan pixel size was $1.52 \mathrm{~nm}$, which has been reduced to $3.8 \AA$ through upsampling when the image shifts are applied.

tcBF-STEM has been applied to thick, whole-cell specimens for which the elimination of chromatic aberration through STEM imaging gives vastly improved images compared to TEM where chromatic aberration causes blurring, or zero-loss EFTEM, where the electrons that contribute to blur are removed resulting in low signal from the thick regions [4]. Improved information transfer was observed in whole $E$. coli cells using the bright field signal, which in the 
image shown in Figure $2 \mathrm{a}$ amounts to only $\sim 35 \%$ of the incident dose (Figure $2 \mathrm{~b}$ ). In thinner specimens such as ribosomes embedded vitreous ice many more electrons are scattered into the bright field region: in the area shown in Figure 1a, $80 \%$ of the incident beam is contained in the bright-field disk. The additional signal in the bright field region compared to cellular specimens should allow similar quality imaging with smaller incident dose, important for preserving highresolution structural information. [5]

[1] Y. Jiang, et al, arXiv:1801.04630 (2018)

[2] P. M. Pelz, et al, Scientific Reports 7 (2017) p. 9883.

[3] M. W. Tate, et al, Microscopy and Microanalysis 22 (2016) pp. 237-249.

[4] K. A. Spoth, et al, Microscopy and Microanalysis 23 (2017) pp. 804-805.

[5] Work supported by the Packard Foundation and NSF awards (DMR-1429155 and DMR1719875).
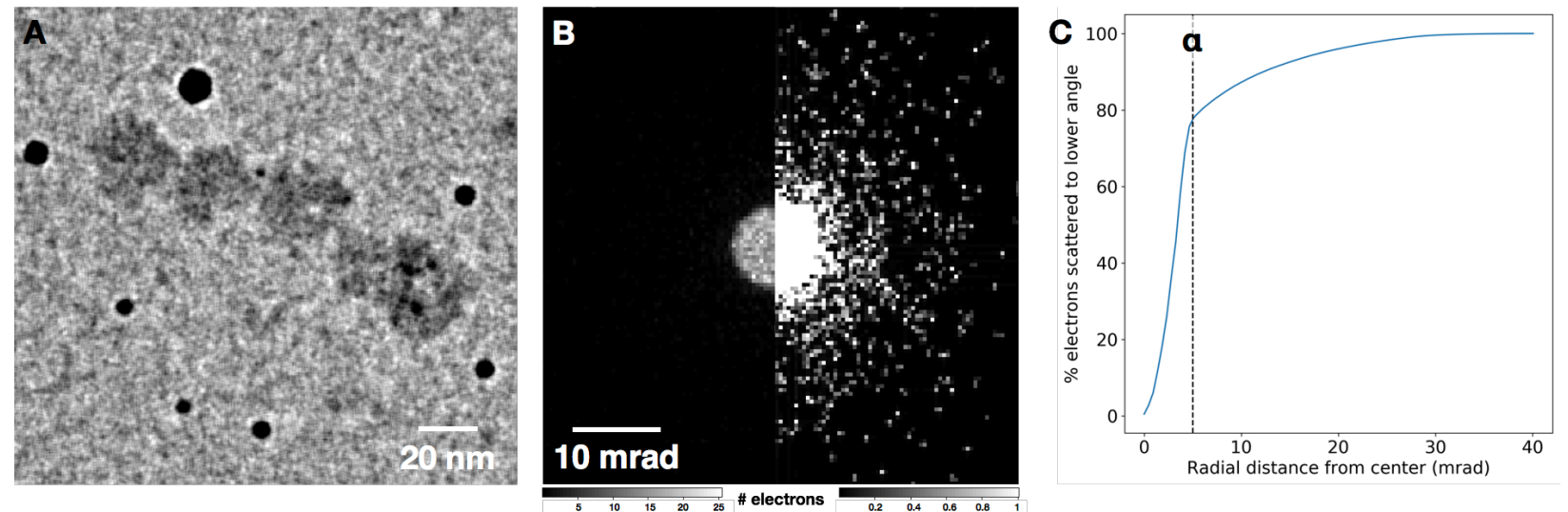

Figure 1. Ribosomes imaged using tcBF-STEM on the EMPAD. (a) Tilt-corrected image, dose rate $\sim 30 \mathrm{e}^{-} / \AA^{2}$. Dark regions are gold fiducials used for focusing. (b) One ribosome CBED imaged on the EMPAD, with contrast scaled to show detail in BF disk (left) as well as singleelectron events at high angles (right). (c) The majority of incident electrons are used to form the tcBF-STEM image of ribosomes.
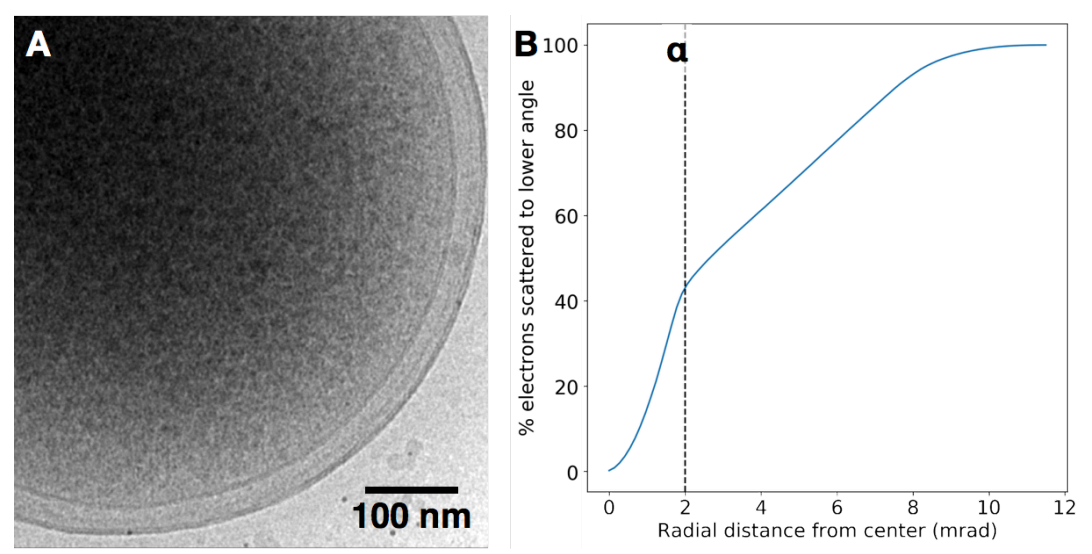

Figure 2. tcBF-STEM imaging of E. Coli. (a) The technique shows good performance in thick specimens where TEM suffers from chromatic aberrations. (b) Most electrons scatter out of the bright field disk in thick specimens; here $\sim 35 \%$ of incident electrons contributed to the image. 